\title{
Special Issue Editorial: Advances in Computational Intelligence and Applications
}

\author{
Zhigang Zeng • Haibo He
}

Published online: 26 October 2010

(C) Springer Science+Business Media, LLC 2010

The Seventh International Symposium on Neural Networks (ISNN 2010) was held on June 6-9, 2010 in Shanghai, China. The ISNN 2010 was a great success and provided a high-level international forum for scientists and engineers to present the latest research in neural networks, computational intelligence, cognitive systems, and related fields. To highlight the success of this conference, we edited this special issue for Cognitive Computation. We chose nine papers from over 591 papers submitted to ISNN 2010, which reflect the high quality of the presentations at the conference while capturing the spirit of our theme, "Advances in Computational Intelligence and Applications," for this special issue.

Our goal for this special issue is to present the latest research developments in computational intelligence with a focus on cognitive computation and their applications across a wide range of domains. Over the past decades, we have witnessed tremendous interest and developments in all aspects of computational intelligence research, ranging from both theoretical foundations, principles, to practical applications in different domains. To reflect a flavor of recent research activities in the community, we carefully selected these nine papers for this special issue.

\footnotetext{
Z. Zeng $(\bowtie)$

Department of Control Science and Engineering,

Huazhong University of Science and Technology,

Wuhan 430074, P. R. China

e-mail: zgzeng@gmail.com

H. $\mathrm{He}$

Department of Electrical, Computer, and Biomedical

Engineering, University of Rhode Island,

Kingston, RI 02881, USA

e-mail: he@ele.uri.edu
}

The selected papers can be organized into the following four coherent sections.

The first section is directly related to neural network models and applications. The paper by Deng presents a new control strategy for the chaotic neural network, in which the refractoriness is tuned by using feedback control based on online averaging of network states. Simulation results demonstrate that the proposed model can achieve favorable performance in handling noisy, incomplete, and composite patterns, while also achieving either enhanced or comparable memory capacity compared with the classic Hopfield net and other chaotic neural network models. In the other paper, Gluge et al. discuss recurrent networks for implicit learning of temporal sequences, a very important area to understand high-level cognitive intelligence. The authors propose a recurrent network with backpropagation training algorithm that is adapted to the reinforcement learning scheme. The simulation results fit quantitatively as well as qualitatively to the behavioral results, suggesting the role of temporal context in associative learning scenarios.

The second section is related to language processing and understanding, an important topic in cognitive science and computational intelligence. The paper by Murata et al. investigates the maximum entropy (ME) approach for natural language processing (NLP) problems including machine translation and information extraction. Comparative studies of the proposed approach with the existing techniques are illustrated in this paper. In related work to this field, the paper by Kaznatcheev investigates connectionist models on the interplay of nouns and pronouns in personal pronoun acquisition, a very interesting and important problem in psychology. For instance, the author discusses the learning in the shifting reference situation for children. It is reported that learning of two different noun-and-pronoun addressee patterns is consistent with 
naturalistic studies, while increasing the amount of exposure to noun-patterns can decrease or eliminate reversalerrors in children. This study provides useful suggestions about how the connectionist models can play an important role in such domains.

The third section is related to pattern recognition, a longstanding research focus in the community. We have selected three papers on this topic. The paper by Subirats et al. presents a C-Mantec constructive neural network algorithm for the multi-class classification problem. This algorithm integrates new features such as competition between neurons and a built-in filtering stage for noisy examples. Three different approaches for multi-class problems are considered, including One-Against-All (OAA), One-Against-One (OAO), and P-against-Q (PAQ). Various benchmarks are used to demonstrate the effectiveness of this approach. In the other paper, Liu present a study of using statistical features on the modified discrete cosine transform (MDCT) for support vector machine (SVM) based learning algorithms. Simulation results illustrate the effectives of this approach. Finally, the paper by Tay et al. presents quantum associative networks with overcomplete Gabor wa velet for face recognition. This work reports some interesting analysis of sparse representations for natural scene analysis.

In the last section, we selected two papers to reflect a flavor of recent application developments of computational intelligence techniques. In the paper by Zheng, multimodal biometrics analysis is studied for Palmprint authentication. The proposed method is based on Shape of
Gaussian (SOG) matching by fusing the Palmprint mixedphase features (PMPF) and the Palmprint directional valley features (PDVF). Experimental results illustrate the effectiveness of the proposed approach. In the other paper, Liu present a cognitive diagnosis method: the model-based diagnosis (MBD), for fault diagnosis in traction power supply systems. While many of the existing diagnosis systems are based on expert-system techniques, this method presents a new perspective on how to develop more cognitive-oriented diagnosis approaches for practical applications.

In summary, we hope the selected papers in this special issue have demonstrated some of the latest research developments and achievements as presented at the ISNN 2010 and will motivate future research directions in this interdisciplinary field.

In closing of this editorial, we would like to express our gratitude to many reviewers who helped us in the peerreview process for this special issue. Their expertise guaranteed the high qualify of the selected papers. We would also like to thank Dr. Amir Hussain, the EditorIn-Chief of Cognitive Computation, for his guidance and suggestions in bringing the special issue to the audience. Finally, we would like to sincerely thank the authors for their great ideas and hard work to make this special issue possible.

We hope you will enjoy reading this special issue.

Zhigang Zeng and Haibo He

Guest Editors 